\title{
O equilíbrio estelar e a existência de uma massa máxima para as estrelas
}

\author{
Stellar equilibrium and the existence of a maximum mass of stars \\ M. Bandecchi ${ }^{1}$, J.E. Horvath ${ }^{*}\left[\right.$, P.S. Bretones ${ }^{2}$ \\ ${ }^{1}$ Universidade de São Paulo, Instituto de Astronomia, Geofísica e Ciências Atmosféricas, Departamento de Astronomia, São \\ Paulo, SP, Brasil \\ ${ }^{2}$ Universidade Federal de São Carlos, Departamento de Metodologia de Ensino, São Carlos, SP, Brasil
}

\begin{abstract}
Recebido em 31 de Agosto, 2018. Revisado em 19 de Novembro, 2018. Aceito em 20 de Novembro, 2018.
As condições simultâneas de equilíbrio Virial e equilíbrio hidrostático determinam a existência de soluções para os modelos estelares mais simples. Utilizando estes dois conceitos mostramos que é possível caracterizar o fim da seqüência estelar observando o comportamento da pressão do gás e a radiação, e apontamos que este valor coincide dentro das incertezas com a máxima massa diretamente inferida para estrelas reais.

Palavras-chave: equilíbrio Virial, estrelas, equilíbrio hidrostático.
\end{abstract}

The simultaneous occurrence of Virial and hydrostatic equilibrium determine the existence of solutions for the simplest stellar models. Using these concepts we show how it is possible to characterize the end of the stellar sequence by observing the behavior of the gas and radiation pressures, and point out that the extracted value coincides, within uncertainties, with the maximum mass directly inferred for actual stars.

Keywords: Virial equilibrium, stars, hydrostatic equilibrium.

Com a exceção do Sistema Sol-Terra-Lua, Sistema Solar e temas correlatos, e apesar do seu grande potencial didático e formativo, os temas de Astronomia e Astrofísica raras vezes chegam de forma concreta e extensiva à sala de aula. Existe certamente um atraso secular entre o desenvolvimento real das disciplinas, sua transposição didática e sua utilização efetiva nas escolas contemporâneas. Muitas vezes os professores declaram que, embora tenham interesse em apresentar estes temas aos alunos, a sua própria formação e a indisponibilidade de material adequado são fatores contra a possibilidade de lecionar sobre o tema. Em particular, a formação inicial e continuada de professores tem sido discutida [1] com relação à ausência de disciplinas de-Astronomia.

No que se refere aos documentos curriculares oficiais, os PCN [2], mesmo dirigidos ao Ensino Fundamental mencionam a evolução das estrelas, anãs brancas, gigantes vermelhas, estrelas de nêutrons e buracos negros. Também a Proposta Curricular do estado de São Paulo [3] apresenta, nos conteúdos da disciplina de Física, na primeira série do Ensino Médio, o tema "As etapas da evolução estelar (formação, gigante vermelha, anã branca, supernova)"(2014), os itens mencionados não aparecem de maneira explícita, sendo ausente qualquer explicação sobre a formação, a evolução e o fim das estrelas e sobre os aspectos físicos envolvidos. Livros didáticos [4] ou pa-

*Endereço de correspondência: foton@iag.usp.br. radidáticos [5] também mencionam tais conteúdos para o Ensino Fundamental.

Ainda na pesquisa em educação em Astronomia, são poucos os trabalhos que tratam da Astrofísica Estelar na educação básica como tema principal, tanto no âmbito nacional quanto no internacional. Trabalhos iniciais sobre levantamento de pesquisas na área já demonstraram o baixo número de publicações sobre estrelas como se observa na Revista Brasileira de Ensino de Física, e no Caderno Brasileiro de Ensino de Física [6, 7]. Posteriormente, um levantamento sobre as edições da Revista Latino-Americana de Educação em Astronomia (RELEA) nos primeiros 10 anos [8] aponta que apenas 2 artigos $(2,7 \%)$ abordaram o tema.

No âmbito internacional, Lelliott e Rollnick [9] mencionam em um levantamento de artigos publicados em periódicos internacionais que os temas sobre Terra, gravidade, ciclo dia/noite, estações e Sistema Sol-Terra-Lua são os mais frequentes e poucos são os estudos relacionados às estrelas. Segundo Pasachoff [10], até mesmo no ensino das universidades é possível perceber esse fato. Alguns trabalhos [11-14] que fazem levantamentos sobre concepções de alunos sobre as propriedades e formação de estrelas já foram publicados. Como recursos didáticos, Reinfeld e Hartman [15] propõem atividades cinestésicas com estudantes para a compreensão do ciclo de vida das estrelas e Futurani [16] um modelo tridimensional das estrelas. 
Além disso, algumas dissertações de mestrado fizeram estudos sobre o tema. Sobre o ensino de astronomia estelar na formação de professores de Ciências na Colômbia, podemos citar o trabalho de Polanco Erazo [17]. Também, mais recentemente no Brasil, Bandecchi [18] apresenta, aplica e analisa uma proposta de ensino de conteúdos referentes à astrofísica estelar para alunos da $2^{\mathrm{a}}$ série do Ensino Médio, abordando os temas: luz, gravidade, temperatura, composição, fusão nuclear, e equilíbrio hidrostático através do ciclo evolutivo das estrelas, desde sua formação até o estágio final.

Outro fator importante é a ausência de aplicações astrofísicas para os conceitos básicos dos cursos de Física, pois apresentam os melhores exemplos e ricas conexões que poderiam contribuir bastante para esta formação. Somente a modo de exemplo, dificilmente os estudantes se defrontam com a radiação cósmica de fundo (CMBR) como o corpo negro mais perfeito medido na natureza (os desvios estão na quinta casa decimal e são estudados para compreender a formação de estrutura no Universo...). Da mesma forma, uma série de conceitos fundamentais na Mecânica dos Fluidos encontra sua melhor aplicação no estudo das estrelas, realização física desta teoria que pode servir como exemplo claro do seu funcionamento e seus alcances. Com efeito, no estudo das estrelas devemos colocar para funcionar a maquinaria completa da Mecânica e outros ramos da Física, tais como o Eletromagnetismo, se quisermos atingir um grau mais profundo de entendimento [19]. Essencialmente toda a Física do século 20 está em ação e podemos dizer que o desenvolvimento da Teoria da Evolução e Estrutura Estelares é a síntese mais cabal da extensão destes estudos, e prova do estado-da-arte da mesma quando confrontada com os dados disponíveis. Como passo prévio para uma descrição completa, as estrelas devem ser entendidas como fluidos auto-gravitantes em equilíbrio, e deste conceito aparentemente simples nos ocuparemos a seguir.

Os modelos estelares mais simples, esfericamente simétricos e desprovidos de toda complicação real (campo magnético, etc.) fornecem um embasamento sólido para entender a estrutura e evolução das estrelas. Estes modelos resultam da solução simultânea das quatro equações diferenciais que relacionam os "gradientes" $\frac{d P}{d r}, \frac{d M}{d r}, \frac{d T}{d r} \mathrm{e}$ $\frac{d L}{d r}$ no interior com as variáveis relevantes temperatura $\stackrel{d r}{T}$, densidade $\rho$ etc. e quantidades constitutivas tais como a opacidade média $\bar{\kappa}$ e outras. Para nossos propósitos, a mais fundamental (no sentido de estabelecer o estado mecânico do gás) destas equações é a equação de equilíbrio hidrostático para uma esfera auto-gravitante

$$
\frac{d P}{d r}=-\frac{G m \rho}{r^{2}}
$$

onde $m(r)$ é a massa encerrada no raio $r$. A solução da equação de equilíbrio hidrostático em termos da função pressão $P(r)$ permite ver que a estrela está, em toda sua extensão radial, sujeita ao balanço de forças de pressão e a gravitação $\sum P_{i}=$ " $P_{\text {grav }}$ ", onde $P_{\text {grav }}=-\frac{\partial E_{\text {grav }}}{\partial V}$ é uma forma de escrever uma quantidade com dimensões de pressão decorrente do campo gravitacional. Esta última forma pode ser vista como algo puramente formal, e por isso a escrevemos entre aspas, mas resulta muito geral, pois a pressão sempre pode ser pensada como o efeito de variar a energia a respeito do volume que esta ocupa [20].

Porém, no problema do equilíbrio estelar existe uma relação "esquecida"de grande importância, que indica como a matéria estabelece a partilha entre a energia interna $E_{\text {int }}$ e a gravitacional $E_{\text {grav }}$, no chamado equilíbrio Virial

$$
E_{\text {grav }}+2 E_{\text {int }}=0
$$

Sabemos, de fato, que o equilíbrio Virial precede o estabelecimento do equilíbrio hidrostático. Existem vários exemplos de estruturas onde o equilíbrio Virial é satisfeito, mas não estão em equilíbrio hidrostático. As próprias estrelas satisfazem esta condição a caminho da Seqüência Principal: a contração da nuvem-mãe está em equilíbrio Virial, mas não atinge o equilíbrio hidrostático até que o colapso seja detido pela pressão interna.

Para formalizarmos a ideia de como este duplo equilíbrio determina o comportamentoda estrela, cabe agora escrevermos explicitamente a $P_{\text {grav }}$ anteriormente definida no caso mais simples. Para uma esfera homogênea, a energia gravitacional em termos do volume $V$ é

$$
E_{\text {grav }}=-\frac{3}{5}\left(\frac{4 \pi}{3}\right)^{1 / 3} \frac{G M^{2}}{V^{1 / 3}}
$$

E assim temos de imediato

$$
P_{\text {grav }}=C M^{2 / 3} \rho^{4 / 3}
$$

$\operatorname{com} C=\frac{G}{5}\left(\frac{4 \pi}{3}\right)^{1 / 3}$.

O membro que contém as propriedades da matéria e a radiação $\sum P_{i}$, por sua vez, pode ser escrito como a soma dos termos de gás e radiação, ambos funções de $\rho$ e T. Para determinar o problema em geral, é necessário obter uma relação entre a pressão e a densidade chamada de equação de estado, na qual no caso mais simples depende de forma complexa destas varáveis. Porém, a equação de estado pode ser grandemente simplificada para um caso especial muito relevante: quando a componente da radiação é pequena e o transporte é totalmente convectivo.

Este problema foi considerado pela primeira vez por Lord Kelvin, na forma de uma estrela em equilíbrio convectivo adiabático, isto é, onde bolhas de fluido sobem e descem sem trocar calor com o ambiente. Da Termodinâmica elementar, sabemos que uma trajetória adiabática nessas condições pode ser descrita como $P=K \rho^{\gamma}$, com $\gamma=5 / 3$ e $V=1 / \rho$ para um gás ideal.Mais precisamente, se o equilíbrio térmico se mantém ponto a ponto, isto quer dizer que a pressão e a densidade devem satisfazer na estrela a mesma relação, e assim Lord Kelvin chegou a considerar esta relação que tem a mesma forma funcional de uma politrópica de índice $3 / 2$. O raciocínio é o 
seguinte: a componente dominante da pressão no interior estelar é a do gás ideal, portanto

$$
P=P_{g a s}=\frac{\rho}{\bar{\mu} m} k_{B} T \Rightarrow T \propto \frac{P}{\rho}
$$

A hipótese convectiva de Lord Kelvin pode ser escrita usando o critério de instabilidade convectivo (formulado por Scwharzschild), segundo o qual se o gradiente de temperatura supera um valor limite $\nabla_{a d}$, a convecção é mais eficiente para transportar energia e o movimento do fluido [21]. O valor desse gradiente adiabático é

$$
\nabla_{a d}=\frac{P}{T} \frac{d T}{d P}=\left(\frac{\partial \ln T}{\partial \ln P}\right)
$$

Para um dado valor constante do expoente podemos integrar a eq. 6. e obter a relação pressão-temperatura de imediato, a qual resulta

$$
P \propto T^{1 / \nabla_{a d}}
$$

Combinando as eqs. (5) e (7), temos de imediato que

$$
P^{1-1 / \nabla_{a d}} \propto \rho^{-1 / \nabla_{a d}} \Rightarrow P=K \rho^{\frac{1}{1-\nabla_{a d}}}
$$

com $K$ independente da microfísica. Então, quando a estrela inteira é suposta em convecção, chegamos à chamada solução de Jeans, e ela implica que as trajetórias adiabáticas do gás satisfazem $P=K \rho^{5 / 3}$, já que para um gás monoatômico totalmente ionizado o valor do expoente é $\gamma=5 / 3 \mathrm{e}$ o gradiente adiabático é simplesmente $\nabla_{a d}=\frac{\gamma-1}{\gamma}=\frac{2}{5}$.

Os cálculos detalhados mostram que as estrelas de $\sim 20 M_{\odot}$ já são quase totalmente convectivas [21]. Este valor está bem abaixo da massa máxima esperada, e portanto a soma $P_{\text {total }}=P_{\text {gas }}+P_{\text {rad }}$ ainda é dominada pelo termo do gás para estrelas observadas, e isto resulta em uma potência de $\rho$ com expoente diferente da $P_{\text {grav }}$. A inclinação da $P_{\text {gas }}$ é maior (o expoente é próximo de $5 / 3$ ), e existe sempre um ponto onde $P_{\text {total }} \approx P_{\text {gas }}=$ $P_{\text {grav }}$, ou seja, uma solução da equação de equilíbrio hidrostático que representa uma estrela (vide Fig. 1).

Podemos agora discutir o problema da existência de uma massa estelar máxima usando estas ideias. Pela

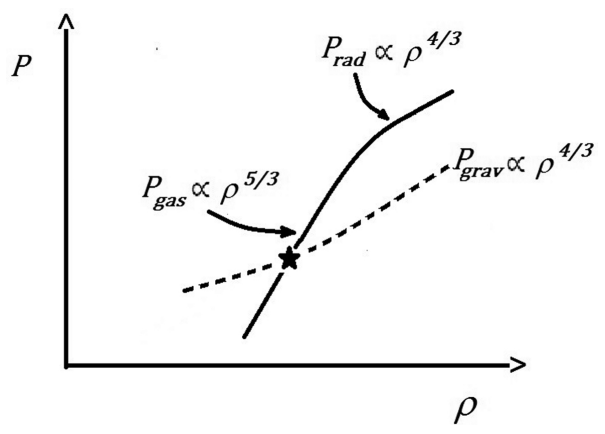

Figura 1: O equilíbrio hidrostático estelar expressado graficamente como a intersecção das pressões $P_{\text {gas }}$ e $P_{\text {grav }}$. mesma razão que a Física determina a estabilidade de qualquer estrutura, as estrelas não podem ter massas arbitrariamente grandes, e embora há discussão intensa na literatura especializada (vide abaixo), não há observações que indiquem a presença de estrelas com massas maiores que $\sim 100-200 M_{\odot}$. Isto sugere que a Física básica do equilíbrio contém razões para que a estrutura estelar acabe em torno desse valor.

Como mostrado na figura 1 , o ponto de intersecção (estrela) das linhas cheia e pontilhada $P_{\text {total }} \approx P_{\text {gas }}=$ $P_{\text {grav }}$ define a existência de uma estrela para massa fixa dada. Quando massas maiores forem consideradas, a curva sólida começa a ter uma contribuição crescente da componente de radiação e fica assintoticamente paralela à $P_{g r ? ? v}$. Assim, as soluções (estrelas) deixam de existir.

Desta forma, sabemos que quando $M \rightarrow M_{\max }$ a componente da radiação rapidamente ganha importância, e finalmente $P_{\text {tot }} \rightarrow P_{\text {rad }}=\frac{1}{3} a T^{4}$. Mas como a estrela precisa satisfazer a relação Virial eq.(1), podemos escrever explicitamente que

$$
\frac{G M^{2}}{R} \sim \frac{N_{o}}{\bar{\mu}} k_{B} T M \Rightarrow T \sim \frac{G \bar{\mu}}{N_{o} k_{B}} M^{2 / 3} \rho^{1 / 3}
$$

Na aproximação $\rho=$ cte (suficientemente acurada para nossos propósitos), vemos que o quociente $T^{3} / \rho$ na estrela se mantém constante sempre, e assim $P_{\text {rad }}=\frac{1}{3} a T^{4}$ implica também que $P_{r a d} \propto \rho^{4 / 3}$. Quando a massa estelar é grande o suficiente, a curva da pressão total começa a ser dominada pela pressão de radiação, e sua inclinação muda para ficar cada vez mais paralela à $P_{\text {grav }}$, conforme aumentarmos a massa estelar. Assim, as soluções deixam de existir em algum ponto pouco antes que o paralelismo completo aconteça, para alguma massa da ordem de $\sim 100-200 M_{\odot}$ (onde a $P_{\text {gas }}$ é dominada pela $P_{\text {rad }}$ ) como conseqüência direta da manutenção estrita da relação Virial.

O confronto destas ideias com as observações pode ser apreciado na figura 2. Três das maiores massas já determinadas são mostradas no diagrama HR. A estrela R136a1 é ao presente a recordista, com massa estimada de $\sim 320 M_{\odot}$ (quando descoberta pensava-se que esse sistema triplo era uma única estrela com $\sim 1000 M_{\odot}$, mas hoje sua natureza múltipla é indiscutida). AG Car com massa $\sim 100 M_{\odot}$ e WR102ka cuja estimativa é de $\sim 150 M_{\odot}$ completam o grupo. Existem mais algumas candidatas detectadas, mas é importante destacar que, devido à existência de ventos que levam gás da estrela e outros fatores que complicam a análise, qualquer valor de massa acima de $\sim 150-200 M_{\odot}$ é tido como controverso. De qualquer forma é um fato que estas estrelas que têm luminosidades $\sim$ milhões de vezes maiores que a do Sol validam as ideias teóricas descritas anteriormente.

Pelo fato que temos ignorado outros vários fatores, é possível que a massa máxima real seja até um pouco menor do que corresponde àquela onde a radiação domina absolutamente, mas o importante é que este exercício mostra que a estrutura estelar existe como decorrên- 


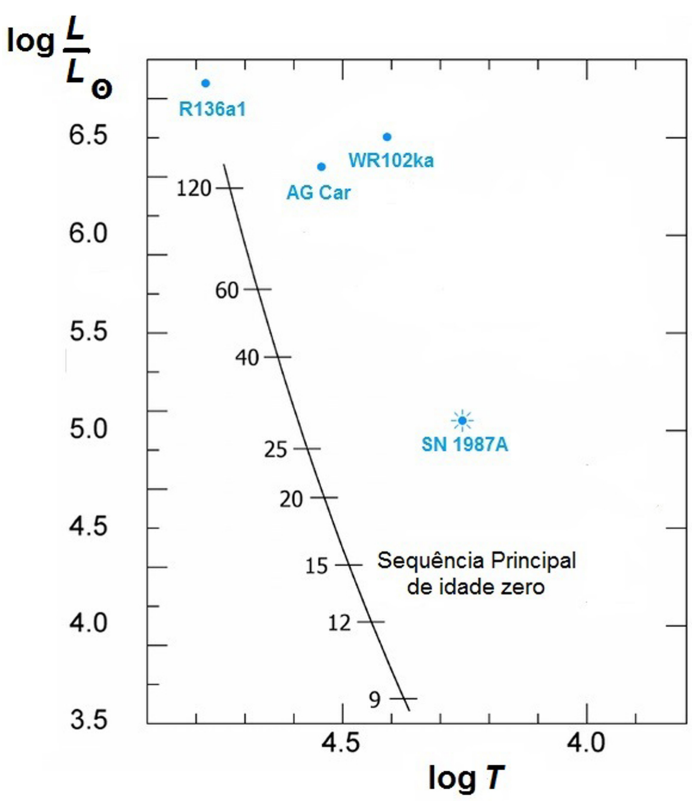

Figura 2: A posição de algumas das estrelas mais massivas no diagrama HR. Para efeitos de comparação, a Seqüência Principal de idade zero com os valores das massas iniciais calculados teoricamente se mostram na linha preta à esquerda. O progenitor da supernova 1987A cuja massa inicial é estimada em $\sim 18-19 M_{\odot}$ aparece na parte inferior.

cia do equilíbrio Virial, fato que raramente é enfatizado o suficiente. Podemos dizer que o Teorema do Virial adquire um caráter concreto que beneficia em muito a compreensão do seu significado, mais ainda tendo como corolário a existência de uma massa máxima para as estrelas. O argumento físico simples apresentado mostra por quê há estrelas, e que alguma massa máxima para elas deve existir. Sugerimos que isto constitui um assunto importante para ser discutido em sala de aula no Ensino Superior, e onde este recurso didático pode ser aplicado. O fato de que a pressão da radiação cumpre um papel desestabilizador da estrutura estelar não deve ser minimizado. Em um número expressivo de casos os estudantes e seus professores acreditam que a radiação tem um papel fundamental na Estrutura Estelar, quando na verdade acontece exatamente o contrário, somente quando esta última não é importante é que existem as estrelas. Esta discussão constitui um complemento importante para o assunto aqui apresentado.

\section{Referências}

[1] P.S. Bretones, Disciplinas Introdutórias de Astronomia nos Cursos Superiores do Brasil. Dissertação de Mestrado, Universidade Estadual de Campinas (1999).

[2] BRASIL, Parâmetros Curriculares Nacionais: Ensino Médio (Ministério da Educação. Brasília, 1998).

[3] SÃO PAULO, Currículo do Estado de São Paulo: Ciências Humanas e suas tecnologias (Secretária da Educação, São Paulo, 2011).
[4] S. Sasson, C. Silva Júnior e P.S.B. Sanches, Ciências. Entendendo a Natureza. $6^{\circ}$ Ano (Ed. Saraiva, São Paulo, 2013), $25^{\mathrm{a}}$ ed.

[5] P.S. Bretones, Os Segredos do Universo (Ed. Atual, São Paulo, 2014), 11 ${ }^{\mathrm{a}} \mathrm{ed}$

[6] J. Marrone Júnior e R.H. Trevisan, Caderno Brasileiro de Ensino de Física 26, 547 (2009).

[7] G. Iachel e R. Nardi, Ensaio Pesquisa em Educação em Ciências 12, 225 (2010).

[8] P.S. Bretones, L.C. Jafelice e J.E. Horvath, Journal of Astronomy \& Earth Sciences Education 3, 110 (2016).

[9] A. Lelliott e M. Rollnick, Int. Jour. Sci. Edu. 32, 13 (2010).

[10] J.M. Pasachoff, Astron. Edu. Rev. 1, 124 (2002).

[11] L. Agan, Astron. Edu. Rev. 3, 77 (2004).

[12] J.M. Bailey, Astron. Edu. Rev. 6, 133 (2007).

[13] J.M. Bailey, E.E. Prather, B. Johnson e T.F. Slater, Astron. Edu. Rev. 8, 2 (2009).

[14] G. Iachel, Revista Latino-Americana de Educação em Astronomia 12, 7 (2011).

[15] E.L. Reinfeld e M.A. Hartman, Astron. Edu. Rev. 7, 158 (2008).

[16] T. Futurani, Astron. Edu. Rev. 7, 122 (2008).

[17] Y. Polanco Erazo, Enseñanza de astronomía estelar a docentes en formación en ciencias naturales. Dissertação de Mestrado, Universidad del Valle, Cali (2016).

[18] M.B.F. Vieira, Astrofísica estelar no Ensino Médio: análise de uma proposta. Dissertação do Mestrado, Universidade de São Paulo (2018).

[19] J.E. Horvath, Rev. Bras. Ens. Fís. 35, 1 (2013).

[20] A. Sommerfeld, Lectures on Theoretical Physics, Volume 5: Thermodynamics and Statistical Mechanics (Academic Press, New York, 1964)

[21] J.E. Horvath, Fundamentos de Evolução Estelar, Supernovas e Estrelas Compactas (Ed. Livraria da Física, São Paulo, 2011). 